\title{
Causal attribution fractions for epidemiological studies, applied to a UK Biobank study of smoking and BMI
}

\author{
Anthony J. Webster
}

Nuffield Department of Population Health, Big Data Institute, Old Road Campus, University of Oxford, Oxford, OX3 7LF, UK.

Epidemiological studies often use proportional hazard models to estimate associations between potential risk factors and disease risk. It is emphasised that when the "backdoor criteria" from causal-inference applies, if diseases are sufficiently rare, then the proportional hazard model can be used to estimate causal associations. When the "frontdoor criteria" applies (allowing causal estimates with unmeasured confounders), similar estimates are found to mediation analyses with measured confounders. Reasons for this are discussed. An attribution fraction is constructed using the average causal effects (ACE) of exposures on the population, and simple methods for its evaluation are suggested. It differs from the attribution fraction used by the World Health Organisation (WHO), except for specific circumstances where the latter can agree or provide a bound. A counterfactual argument determines an individual's attribution fraction $A_{f}$ in terms of proportional hazard estimates, as $A_{f}=1-1 / R$, where $R$ is an individual's relative risk. Causally meaningful attribution fractions cannot be constructed for all known risk factors or confounders, but there are important cases where they can. As an example, systematic proportional hazards studies with UK Biobank data estimate the attribution fractions of smoking and BMI for 226 diseases. The attribution of risk is characterised in terms of disease chapters from the International Classification of Diseases (ICD-10), and the diseases most strongly attributed to smoking and BMI are identified. The result is a quantitative characterisation of the causal influence of smoking and BMI on the landscape of disease incidence in the UK Biobank population.

\section{Introduction}

The original aim of this research was to quantify the modifiability of disease risks by several behaviours and physical characteristics, such as smoking and body mass index (BMI). Risks are often quantified with attributable fractions and relative risks [1, 2], that are estimated using proportional hazard models [2, 3]. However, there are several ways of defining and estimating attributable fractions [2, 4, 5], and estimates of relative risks do not generally have a causal interpretation [2].

The recently established theory of causal inference $[6,7]$ is used to identify common situations where relative risk estimates from proportional hazard models provide estimates of causal associations. These can then be used to form attributable fractions that characterise the causal influence of exposures on disease risk. A causal analysis limits the exposures whose associations can be given a causal interpretation. Fortunately these included smoking and BMI, that are widely acknowledged to have a strong influence on a wide range of diseases.

Whereas statistics is the science of finding and describing patterns in data, epidemiology is the science of using statistics to make correct inferences. Although epidemiologists are careful to describe their results in terms of "associations", the purpose of epidemiology is to detect and quantify causal associations, e.g. between lifestyles and health [2, 8]. Recently the science of causal inference $[6,7,9]$, has developed to allow estimates of causal associations to be made, without data from randomised control trials (RCTs). By exploring the relationship between causal estimates that are made with the "backdoor criteria" and the "do" calculus [6,7], and conventional epidemiological estimates of relative risks using proportional hazards [3], it is shown that if formulated and interpreted correctly, many conventional epidemiological studies [2, 3] will correctly estimate causal associations. It is also shown how proportional hazards estimates can be applied to situations that satisfy the "frontdoor" criteria, and how the results can coincide with mediation analyses [9]. An attribution fraction is suggested that uses estimates of causal associations. It is closely related to the average causal effect (ACE) [6, 7], and it can agree with existing expressions when these are combined with estimates of causal associations [1,4]. An attribution fraction for an individual is also formulated using a counterfactual argument that combines the "effect of treatment on the treated" [6, 7] and proportional hazard estimates, that gives a simple and widely used expression in terms of an individual's relative risk.

As an example of the approach with importance for public health policy, the attribution of disease incidence to smoking and BMI was considered for the UK Biobank cohort of over 500,000 UK men and women [10]. Attribution fractions

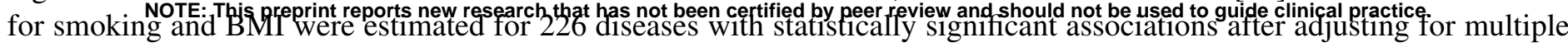


testing, using conventional proportional hazards models that are adjusted for known confounding factors. The results emphasise the heterogeneous influence of risk factors, with protective associations for several diseases. They also emphasise the pathogenic influence of smoking and BMI on some diseases, with 11 having an attributable fraction of 0.5 or greater.

\section{Causal inferences}

\section{Relative risks and the "backdoor criteria"}

Consider how disease risk may be influenced by the established common risk factors of education, socio-economic status, BMI, alcohol, and smoking (figure 1). It seems likely that for many diseases, education and socio-economic status could influence disease risk through the modifiable risk factors of BMI, alcohol, and smoking, in addition to any direct risk. In those circumstances education and socio-economic status are confounders (denoted with a vector $Z$ ), that influence both disease risk and the values of BMI, alcohol, and smoking (denoted with a vector $X$ ). For this causal model illustrated in figure 1, it is possible to estimate the consequences of setting BMI, alcohol, and smoking to a specific value $X=x$, corresponding to $\operatorname{do}(X=x)$ using the "do" notation of Pearl [6,7]. The situation corresponds to the well-known situation described by the

(a)

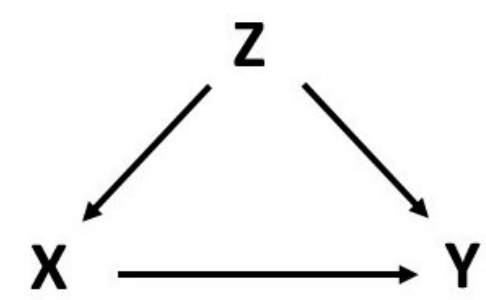

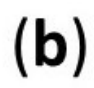

\section{$-\left[\begin{array}{l}\text { Smoking, } \\ \text { BMI, ... }\end{array}\right]$}
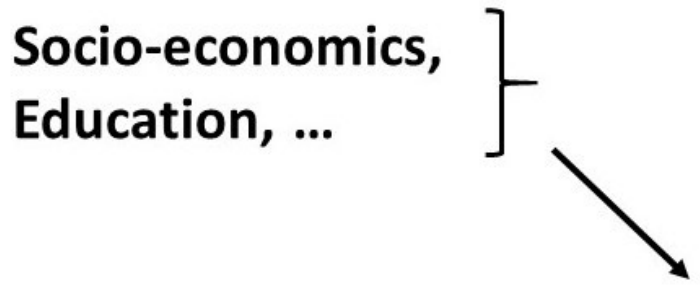

\section{Disease risk}

Figure 1: Consider the influence of one or more exposures $X$, on diseases $Y$, with confounding variables $Z$ that satisfy the "backdoor criteria" [6, 7] (figure a). For example, $X$ might include BMI, alcohol, and smoking, with confounders $Z$ of socio-economic status and education (figure b).

"adjustment" formula, that states,

$$
P(Y=y \mid \operatorname{do}(X=x))=\sum_{z} P(Y=y \mid X=x, Z=z) P(Z=z)
$$

where for continuous variables, the sums are treated as integrals. The formula is constructed to account for the confounding influence of $Z$ on both $X$ and disease risk, and differs from that given by conventional probability theory, that would have $P(Z=z \mid X=x)$ instead of $P(Z=z)$. Take $Y=1$ to denote presence of disease, and $Y=0$ its absence, so that,

$$
P(Y=1, T<t \mid X=x, Z=z)=F(t, x, z)
$$

where $F(t, x, z)$ is the distribution function (with covariates $x, z$ ), so that,

$$
\begin{aligned}
F(t, x, z) & =1-S(t, x, z) \\
& =1-\exp (-H(t, x, z)) \\
& \simeq H(t, x, z) \\
& =e^{\eta_{x}+\eta_{z}} H_{0}(t) \quad \text { with } \quad H_{0}(t)=\int_{0}^{t} h_{0}(s) d s
\end{aligned}
$$

where in going from the 2nd to 3rd line we assume sufficiently rare diseases, as is the case for the first hospital admission for most diseases in UK Biobank [10, 12], and in going from the 3rd to the 4th lines we assume that the proportional hazards assumption [3] is valid for the disease being studied, with $\eta_{x}$ and $\eta_{z}$ being the linear predictor functions ${ }^{1}$ for the (possibly)

\footnotetext{
${ }^{1}$ For a particular $X=x$, the linear predictor function is sometimes referred to as the "linear component", "risk score", or "prognostic index" [3].
} 
multivariate variables $x$ and $z$. For a probability density $f=d F / d t$ and hazard function $h=f / S, H(t)=\int_{0}^{t} h(t)$ is the cumulative hazard, and a proportional hazards model assumes that $h(t, x, z)=h_{0}(t) e^{\eta_{x}+\eta_{z}}$. Now using Eq. 1 ,

$$
\begin{aligned}
& P(Y=1, T<t \mid \operatorname{do}(X=x)) \\
& =\sum_{z} P(Y=y, T<t \mid X=X, Z=z) P(Z=z) \\
& \simeq \sum_{z} e^{\eta_{x}+\eta_{z}} H_{0}(t) P(Z=z) \\
& =e^{\eta_{x}} H_{0}(t) A_{Z}
\end{aligned}
$$

with $A_{Z} \equiv \sum_{z} e^{\eta_{z}} P(Z=z)$. This allows the incidence rates to be calculated for a (possibly hypothetical) situation where we have intervened in some way to set $X=x$, in terms of a baseline hazard function that is estimated in the usual way, using observational data in which $Z$ can be correlated with both $X$ and disease risk. Note that $P(Z=z)$ and $P(X=x)$ are implicitly the population values at the study's start.

At the baseline values of $x=x_{0}$ and $z=z_{0}$, by definition $\eta_{x}\left(x_{0}\right)=0$ and $\eta_{z}\left(z_{0}\right)=0$, so Eq. 4 gives $P(Y=1, T<$ $\left.t \mid X=x_{0}, Z=z_{0}\right)=H_{0}(t)$. Then with the same approximations used to derive Eq. 4, Eq. 4 can be written in several different ways, for example with,

$$
\begin{aligned}
& P(Y=1, T<t \mid \operatorname{do}(X=x)) \\
& =e^{\eta_{x}} A_{Z} H_{0}(t) \\
& =e^{\eta_{x}} A_{Z} P\left(Y=1, T<t \mid X=x_{0}, Z=z_{0}\right) \\
& =A_{Z} P\left(Y=1, T<t \mid X, Z=z_{0}\right)
\end{aligned}
$$

When education and socio-economic factors are represented by $Z$, then the factor $A_{Z}$ accounts for changes in risk due to both socio-economic factors and education, and the influence of setting $X=x$ is calculated through the factor $e^{\eta_{x}}$. If we could set $X$ equal to the baseline values $x_{0}$, the probability distribution would be proportional to the baseline hazard function $H_{0}(t)$, amplified or shrunk by the factor $A_{Z}$. If the baseline values corresponded to the lowest disease risk, then $A_{Z} H_{0}(t)$ would be the lowest possible disease incidence rate that could have been achieved through lifestyle changes. Eq. 5 can be written as,

$$
\frac{P(Y=1, T<t \mid \operatorname{do}(X=x))}{P\left(Y=1, T<t \mid \operatorname{do}\left(X=x_{0}\right)\right)}=e^{\eta_{x}}
$$

This gives a relative risk of disease within time $t$ for a population with $X=x$, compared with a population with baseline values of $X=x_{0}$, in terms of estimates from a relative risk from observational studies, that have,

$$
e^{\eta_{x}}=\frac{h\left(t \mid X=x, Z=z_{0}\right)}{h\left(t \mid X=x_{0}, Z=z_{0}\right)}
$$

Appendix A relates the argument to probability densities and hazard functions.

The analysis above applies more generally to other examples, and to studies other than those involving disease or health. Similar results will apply whenever $F(t, x, z)$ can be factored as $H_{0}(t) g(x) q(z)$, for some functions $g(x)$ and $q(z)$, as was possible here because we consider a proportional hazards model and situations whose the incidence is sufficiently rare that we can approximate $F(t, x, z) \simeq H(t, x, z)$.

\section{Unmeasured confounders and mediation - the "frontdoor criteria"}

Another important result from causal inference, is the "frontdoor criteria" [6, 7]. A well-known example [7] is assessing the influence of smoking on disease risk in the presence of unmeasured confounders that influence both smoking use and disease risk, by using an additional measurement of tar in peoples' lungs (figure 2). Again we consider the adjustment formula for this situation in the limit of rare diseases, as above, and consider the simple specific example with continuous variables for e.g. average number of cigarettes per day and tar content of lungs. Although the estimated incidence rates will differ from those using proportional hazards models, the causal estimate for the influence of smoking on lung cancer, is the same as we might (with hindsight) have anticipated from mediation studies.

For the situation described in figure 2, the "front door" adjustment formula states [6, 7],

$$
P(Y=y \mid \operatorname{do}(X=x))=\sum_{z} \sum_{x^{\prime}} P\left(Y=y \mid Z=z, X=x^{\prime}\right) P\left(X=x^{\prime}\right) P(Z=z \mid X=x)
$$




\section{Unmeasured confounder}

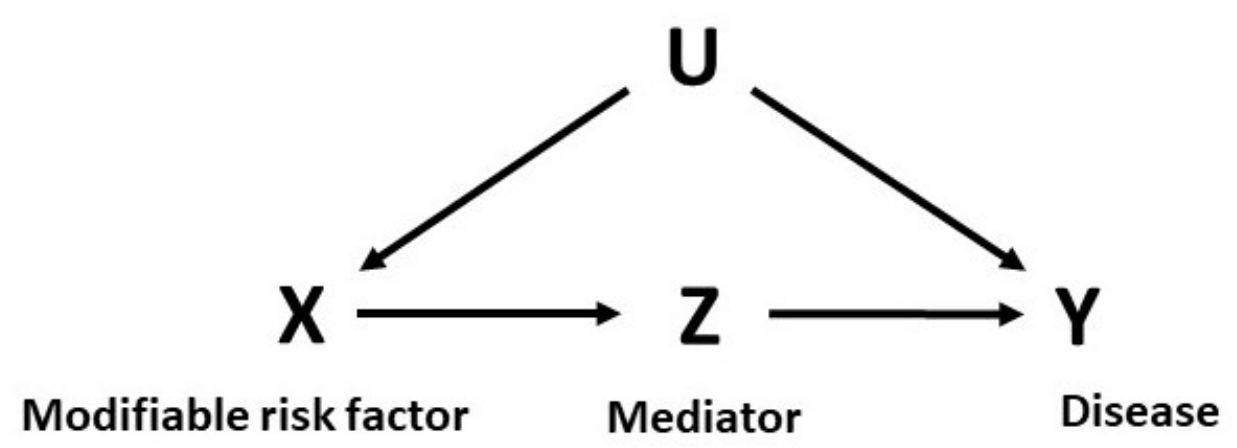

Figure 2: The "frontdoor criteria" estimates the causal influence of an exposure $\operatorname{do}(X=x)$, that is mediated by $Z$, in the presence of unmeasured confounders $U$ that influence both the disease risk and the exposure $X$.

Using this, and proceeding as before,

$$
\begin{aligned}
P(Y=1, T<t \mid \operatorname{do}(X=x)) & =\sum_{z} \sum_{x^{\prime}} P\left(Y=1, T<t \mid X=x^{\prime}, Z=z\right) P(Z=z \mid X=x) P\left(X=x^{\prime}\right) \\
& \simeq \sum_{z} \sum_{x^{\prime}} e^{\eta_{x^{\prime}}} e^{\eta_{z}} H_{0}(t) P(Z=z \mid X=x) P\left(X=x^{\prime}\right) \\
& =H_{0}(t)\left(\sum_{z} e^{\eta_{z}} P(Z=z \mid X=x)\right)\left(\sum_{x^{\prime}} e^{\left.\eta_{x^{\prime}} P\left(X=x^{\prime}\right)\right)}\right.
\end{aligned}
$$

Next consider the specific example where $\eta_{x^{\prime}}=\beta_{x} x^{\prime}, \eta_{z}=\beta_{z} z, P(X=x)$ is a normal distribution $N\left(\mu_{x}, \sigma_{x}^{2}\right)$, and $P(Z=z \mid X=x)$ is a normal distribution $N\left(\alpha x, \sigma_{z}^{2}\right)$, where in the latter case $\alpha$ is a constant and the mean of $z$ is $\alpha x$. Understanding that the sums should be considered as integrals when variables are continuous, then we have,

$$
\sum_{x^{\prime}} e^{\eta_{x^{\prime}}} P\left(X=x^{\prime}\right)=\exp \left(\beta_{x} \mu_{x}\right) \exp \left(\frac{\sigma_{x}^{2} \beta_{x}^{2}}{2}\right)
$$

and,

$$
\sum_{z} e^{\eta_{z}} P(Z=z \mid X=x)=\exp \left(\beta_{z} \alpha x\right) \exp \left(\frac{\sigma_{z}^{2} \beta_{z}^{2}}{2}\right)
$$

giving,

$$
P(Y=1, T<t \mid \operatorname{do}(X=x)) \simeq H_{0}(t) \exp \left(\beta_{x} \mu_{x}\right) \exp \left(\frac{\sigma_{x}^{2} \beta_{x}^{2}}{2}+\frac{\sigma_{z}^{2} \beta_{z}^{2}}{2}\right) \exp \left(\beta_{z} \alpha x\right)
$$

The incidence rate at baseline $X=x_{0}$ is determined by the first three terms, and differs from a proportional hazard estimate that is adjusted by either or both, of $x$ or $z$. The first two terms are equivalent to a proportional hazards estimate with $x$ at the mean exposure $\mu_{x}$ and $z$ at the baseline value, and the third term quantitatively accounts for the spread in values of $x$ and $z$ about their mean values. The influence of $\operatorname{do}(X=x)$, is seen in the last term $e^{\beta_{z} \alpha x}$, with the change in risk being mediated by $z$ in a very simple and intuitive way.

For the situation considered here, where there is solely an indirect effect of the exposure through the mediator, this estimate is the same as for a mediation analysis with measured confounding [9]. Interestingly, in the equivalent mediation analysis with measured confounding, the influence of measured confounding on the estimate ${ }^{2}$, does not appear in the resulting expressions for natural direct, and indirect, effects. This appears to explain the agreement between estimates with measured, and unmeasured confounding - for the model of figure 2 in limit of rare diseases and a proportional hazards model, the estimate is (apparently) unaffected by confounding.

Equation 9 applies to any situation described by figure 2, and the example given can be generalised, e.g. to multivariate normal distributions.

\footnotetext{
${ }^{2}$ For a solely indirect effect, $\gamma_{1}=\gamma_{3}=0$ in Eq. 4.6 on page 101 of [9], and measured confounding is accounted for through the coefficient $\gamma_{4}$, that does not subsequently appear in the equations for natural direct and indirect effects.
} 


\section{Attributable fractions}

Attributable fractions are intended to describe the proportion of disease incidence that is caused by an exposure, or can be avoided by an intervention. They can be defined in several related but distinct ways [2,5]. Here the attributable fraction for the situation described by figure 1 is considered. To allow exploration of the causal influence of a subset $X$, of risk factors $X$ and $W$, the risk factors are considered to be composed of both $X$ and $W$. If $P(Y=1, T<t)$ is the probability of observing a disease at a time $T$, less than $t$, then the average causal effect of risk factors on disease risk in a population compared with baseline risk factors is, $P(Y=1, T<t)-P\left(Y=1, T<t \mid \operatorname{do}\left(X=x_{0}\right)\right)$, and the excess fraction is,

$$
A_{f}=\frac{P(Y=1, T<t)-P\left(Y=1, T<t \mid \operatorname{do}\left(X=x_{0}\right)\right)}{P(Y=1, T<t)}
$$

The numerator of 13 is the average causal effect (ACE) [6] of the risk factors $X$ on the population's disease risk, compared with the baseline values $X=x_{0}$. It is divided by the probability of risk in the population, giving an excess risk fraction, that is referred to here as an attributable fraction. To evaluate this, firstly note that,

$$
\begin{aligned}
P(Y=1, T<t) & =\int d x d w d z P(Y=1, T<t, X=x, W=w, Z=z) \\
& =\int d x d w d z P(Y=1, T<t \mid X=x, W=w, Z=z) P(X=x, W=w, Z=z) \\
& \simeq H_{0}(t) \int d x d w d z e^{\eta_{x}+\eta_{w}+\eta_{z}} P(X=x, W=w, Z=z)
\end{aligned}
$$

where we assumed the data could be described by a proportional hazards model for sufficiently rare diseases, to allow the approximation $F(t) \simeq H(t)=H_{0}(t) e^{\eta_{x}+\eta_{w}+\eta_{z}}$, where $\eta_{x}, \eta_{w}, \eta_{z}$ are linear predictors respectively involving $x, w$, and $z$. Integrals should be replaced by sums for non-continuous variables. $P\left(Y=1, T<t \mid \operatorname{do}\left(X=x_{0}\right)\right)$ can be evaluated similarly, and for the example of figure 1 considered here, we can use the backdoor adjustment formula in the second line below,

$$
\begin{aligned}
P\left(Y=1, T<t \mid \operatorname{do}\left(X=x_{0}\right)\right) & =\int d w d z P\left(Y=1, T<t, W=w, Z=z \mid \operatorname{do}\left(X=x_{0}\right)\right) \\
& =\int d w d z P\left(Y=1, T<t \mid X=x_{0}, W=w, Z=z\right) P(W=w, Z=z) \\
& \simeq H_{0}(t) e^{\eta_{x_{0}}} \int d w d z e^{\eta_{w}+\eta_{z}} P(W=w, Z=z)
\end{aligned}
$$

Therefore, using Eqs. 14 and 15, the excess fraction is given by,

$$
A_{f}=\frac{\int d x d w d z e^{\eta_{x}+\eta_{w}+\eta_{z}} P(X=x, W=w, Z=z)-e^{\eta_{x_{0}}} \int d x d w d z e^{\eta_{w}+\eta_{z}} P(W=w, Z=z)}{\int d x d w d z e^{\eta_{x}+\eta_{w}+\eta_{z}} P(X=x, W=w, Z=z)}
$$

where $e^{\eta_{x_{0}}}=1$ for the baseline variables $x_{0}$. The equation is very similar to conventional expressions for attributable fractions that use relative risks [5], that would give an attributable fraction of $1-1 / R=1-e^{-\eta_{x}}$, where $R$ is the relative risk. However the expression now involves averages over the population, that include potential correlations with the confounding variables. The WHO uses an attributable fraction that is defined as [1],

$$
A_{W}=\frac{\int d x e^{\eta_{x}} P(X=x)-\int d x e^{\eta_{x}} P^{\prime}(X=x)}{\int d x e^{\eta_{x}} P(X=x)}
$$

where $P^{\prime}(X=x)$ is an alternative probability distribution for $X$. If we take $P^{\prime}(X=x)$ to be a delta function centred on $X=x_{0}$, with $e^{x_{0}}=1$, then,

$$
A_{W}=1-\frac{1}{\int d x e^{\eta_{x}} P(X=x)}
$$

which is the same as would be obtained by assuming that $e^{\eta_{x}}$ and $e^{\eta_{w}+\eta_{z}}$ are uncorrelated in Eq. 16. Appendix B shows that the $A_{W}$ provides a lower (upper) bound on $A_{f}$ if $e^{\eta_{x}}$ is positively (negatively) correlated with $e^{\eta_{w}+\eta_{z}}$. In general Eqs. 16 and 17 will differ, and neither should have a causal interpretation unless the causal model satisfies suitable conditions such as those in figure 1 that ensure that causal associations are being estimated.

To estimate the integrals in 16 , note that $E[f(X)]=E\left[(1 / n) \sum_{i=1}^{n} f\left(X_{i}\right)\right]$ and that the variance $\operatorname{Var}\left[(1 / n) \sum_{i=1}^{n} f\left(X_{i}\right)\right]=$ $(1 / n) \operatorname{Var}(f(X)) \rightarrow 0$ as $n \rightarrow \infty$. This allows the integrals to be approximated by a sum over the observed data, which is reasonable if the number of data points is sufficiently large in each level of categorical data considered. For example, in the 
study of UK Biobank described later with nearly 500,000 participants, the smallest category was for current smokers, but this included over 50,000 smokers. With this approximation,

$$
A_{f} \simeq 1-\frac{\sum_{i=1}^{n} e^{\eta_{w_{i}}+\eta_{z_{i}}}}{\sum_{i=1}^{n} e^{\eta_{x_{i}}+\eta_{w_{i}}+\eta_{z_{i}}}}
$$

That might alternately be written as,

$$
A_{f}=1-\frac{1}{\sum_{i=1}^{n} w_{i} e^{\eta_{x_{i}}}}
$$

with,

$$
w_{i}=\frac{e^{\eta_{w_{i}}+\eta_{z_{i}}}}{\sum_{i=1}^{n} e^{\eta_{w_{i}}+\eta_{z_{i}}}}
$$

which shows that the relative risk is weighted by the influence of confounders and other risk factors, but is similar to conventional expressions attributable fractions with $A=1-e^{-\eta_{x_{i}}}$. When there are no confounders or other risk factors than $x$, then the terms in Eq. 21 become 1 , and $w_{i}=1 / n$, so that $\sum_{i=1}^{n} w_{i} e^{\eta_{x_{i}}}$ is then just the average of $e^{\eta_{x_{i}}}$ across the population. The expression makes it clear that if the relative risk $e^{-\eta_{x_{i}}}$ is positively correlated with the relative risks from the confounding and other potential risk factors $e^{\eta_{w_{i}}+\eta_{z_{i}}}$, then $\sum_{i=1}^{n} w_{i} e^{\eta_{x_{i}}}>\sum_{i=1}^{n} e^{\eta_{x_{i}}}$, and the attribution fraction is greater when accounting for the confounding and other potential risk factors.

To compare the attributable risk between setting $X=x_{1}$ and $X=x_{2}$, the equivalent expression to Eq. 19 is,

$$
\frac{P\left(Y=1, T<t \mid \operatorname{do}\left(X=x_{2}\right)\right)-P\left(Y=1, T<t \mid \operatorname{do}\left(X=x_{1}\right)\right)}{P\left(Y=1, T<t \mid \operatorname{do}\left(X=x_{2}\right)\right)}=1-\frac{e^{\eta_{x_{1}}} \sum_{i=1}^{n} e^{\eta_{w_{i}}+\eta_{z_{i}}}}{e^{\eta_{x_{2}}} \sum_{i=1}^{n} e^{\eta_{w_{i}}+\eta_{z_{i}}}}=1-e^{\eta_{x_{1}}-\eta_{x_{2}}}
$$

which is just the conventional result for attributable fraction in terms of the relative risk.

\section{Number of attributed cases}

The proportion of disease cases that are attributed to a risk factor is only important if the disease is sufficiently common. The change in the number of cases of disease can be estimated using the estimated attributable fraction and the number of observed cases of disease. If $N$ is the population size under consideration, and $P \equiv P(Y=1, T<t), P_{0} \equiv P(Y=1, T<$ $\left.t \mid \operatorname{do}\left(X=x_{0}\right)\right)$, then,

$$
A_{f}=\frac{N\left(P-P_{0}\right)}{N P}
$$

If we approximate $N P$ as the observed number of cases in the population being studied $N_{o b s}$, then we can estimate the number of extra (or fewer) cases from the attributable fraction $A_{f}$, with,

$$
N_{A_{f}} \equiv N\left(P-P_{0}\right)=N P \simeq A_{f} N_{o b s}
$$

This gives a simple estimate for the number of cases that are attributable to a risk factor. However, this is the number of attributable cases of hospital admissions, for diseases included by the study's selection criterion - first admissions in an ICD-10 chapter in this paper. This latter estimate could substantially differ from our perception of the number of hospital admissions caused by a specific disease, that could be dominated by sequences of hospital visits, or result from a different original underlying cause. For that reason, attributable fractions are generally a better measure of the causal influence of risk factors on the risk of disease.

If the attributable fraction given by Eq. 19 were negative, then instead of considering $\left(P-P_{0}\right) / P$, an alternative would be to consider $\left(P_{0}-P\right) / P_{0}$. However, provided $A_{f}$ is reasonably small, then the two estimates have approximately the same magnitude, with a change in sign to indicate the direction of effect. Expanding $\left(P_{0}-P\right) / P_{0}$ in terms of $A_{f}=\left(P-P_{0}\right) / P$, gives,

$$
\frac{P_{0}-P}{P_{0}}=-\left(\frac{P-P_{0}}{P}\right) \frac{1}{1-\left(\frac{P-P_{0}}{P}\right)} \simeq-\left(\frac{P-P_{0}}{P}\right)\left(1+A_{f}\right)
$$

Showing that both expressions are approximately equal in magnitude if $A_{f}$ is small. 


\section{Effect of treatment on the treated (ETT)}

An alternative attribution fraction, that is of more interest to clinicians or an individual, is the chance of having avoided a disease if you had not been exposed, but were subjected to the same correlated confounding factors that you would have otherwise experienced. This situation is equivalent to estimating the "effect of treatment on the treated" (ETT) [6,7], but the "treatment" is an exposure to smoking or BMI. For the situation considered here of figure 1, this counterfactual question can be formulated and expressed in terms of observational quantities in a similar way to before. The argument below considers the simpler situation of smokers versus never smoked, or max BMI tertile versus a lower BMI tertile, denoting exposed by $X=x_{1}$ and unexposed by $X=x_{0}$. Using counterfactual notation where $Y_{x_{1}}$ indicates the disease status of (e.g.) smokers, and $Y_{x_{0}}$ the disease status of non-smokers, then the ETT is defined as [6,7],

$$
E T T=\left[Y_{x_{1}}-Y_{x_{0}} \mid X=x_{1}\right]
$$

that can be thought of as estimating the difference between disease risk in smokers and non-smokers, when subjected to the same correlated confounding influences as smokers would experience. Following a previous derivation [7], and incorporating the same proportional hazards assumptions as before, this can be written as,

$$
\begin{aligned}
E T T & =P\left(Y_{x_{1}}=1 \mid X=x_{1}\right)-P\left(Y_{x_{0}}=1 \mid X=x_{1}\right) \\
& =\int P\left(Y=1 \mid W=w, Z=z, X=x_{1}\right) P\left(W=w, Z=z \mid X=x_{1}\right) d w d z \\
& -\int P\left(Y=1 \mid W=w, Z=z, X=x_{0}\right) P\left(W=w, Z=z \mid X=x_{1}\right) d w d z \\
= & H_{0}(t) e^{\eta_{x_{1}}} \int e^{\eta_{w}+\eta_{z}} P\left(W=w, Z=z \mid X=x_{1}\right) d w d z-H_{0}(t) e^{\eta_{x_{0}}} \int e^{\eta_{w}+\eta_{z}} P\left(W=w, Z=z \mid X=x_{1}\right) d w d z
\end{aligned}
$$

where the second term on the second line is usually justified with the backdoor adjustment formula Eq. 1, but corresponds to estimating the probability of disease when $X=x_{0}$ but all other exposures are as they would have been if $X=x_{1}$, and the third line uses the approximation of sufficiently rare diseases that the cumulative distribution function can be approximated by the cumulative hazard. Continuing to take the baseline value $e^{\eta_{x_{0}}}=1$, and dividing by the first term to get an attribution fraction, then gives,

$$
A_{E T T}=\frac{e^{\eta_{x_{1}}}-1}{e^{\eta_{x_{1}}}}
$$

which solely involves the relative risk $R=e^{\eta_{x_{1}}}$ for e.g. smoking status, and is the simplest attribution fraction that occurs in the literature.

Because survival analyses are designed to estimate the influence of risk on an individual, with hindsight, perhaps Eq. 28 should not have been a surprise? Within the proportional hazards model, smoking will modify your risk of disease, independently of whether any other factors also do. From a population perspective, disease risk is determined by the overall combination of exposures, that will usually be correlated. This is why the attribution fraction for the population needs a more careful estimation that accounts for correlations between the exposures and confounding variables.

\section{Attribution of disease incidence to smoking and BMI}

A key requirement for public health policy, is to assess the impact of exposures such as smoking and BMI on health. Depending on the disease, the influence of smoking or BMI can be very different. The associations can change in both magnitude and direction of effect, being harmful for most diseases but protective for some. Their influence is further complicated by the correlation of risk factors with known confounders such as socioeconomic status and education, something that Eq. 19 is intended to account for. To explore the overall influence of smoking and BMI on disease, the attribution of smoking and BMI to the incidence of over 400 hospital diagnosed diseases in men and women was considered using UK Biobank data [10]. The aim was to characterise diseases by their attribution fractions, allowing them to be ranked or classified in terms of their risk modifiability in terms of smoking and BMI. The selection of diseases for study is detailed elsewhere [13], along with further information on the UK Biobank data that was used [10,13]. Although the survival analyses could be done better by studying each disease in detail and formulating the analysis appropriately, the study here accounts for the strongest confounding factors, while allowing a broad survey of the overall influence of smoking and BMI on disease. The causal model was assumed to be as in figure 1, and the analysis is briefly summarised below. 
medRxiv preprint doi: https://doi.org/10.1101/2021.12.24.21268368; this version posted December $25,2021$. The copyright holder for this preprint (which was not certified by peer review) is the author/funder, who has granted medRxiv a license to display the preprint in

All rights reserved. No reuse allowed without permission.

\section{Survival analysis}

The International Classification of Diseases version 10 (ICD-10) [14], classifies disease diagnoses into chapters of related diseases. To minimise the potential for confounding by prior disease, that might modify incidence rates, only the first incidence of disease in each ICD-10 chapter was considered for each individual who lived long enough to experience it. We considered disease diagnoses that were the primary cause of hospital admission and would have passed a threshold of severity to trigger hospital admission, as identified from hospital episode statistics (HES), and recorded with an ICD-10 code. Individuals who reported diabetes at entry to the study were excluded. This ensured that new cases of diabetes would almost entirely involve type II diabetes, with risk most strongly influenced by age and BMI. For each disease studied, data were excluded if the disease occurred before a participant entered the study, or if an individual had a hospital diagnosed cancer other than non-melanoma skin cancer before starting the study. The disease incidence rates are "rare" in the approximate sense needed to estimate attribution fractions [12]. A survival analysis using age as the time variable was left truncated at a participant's entry to the study, right-censored if there was: death, cancer other than non-melanoma skin cancer, or the study period ended. All diagnoses recorded between entering the study and 31st January 2020 were included, as recorded in UK Biobank HES data on 8th December 2021. Data beyond 31st January 2021 were likely to be influenced by the COVID-19 pandemic and were omitted. Analyses were multiply adjusted using a proportional hazards model, with men and women studied separately, and a causal model assumed as in figure 1. Adjustment considered the established risk factors of: smoking status (never, previous, or current), alcohol consumption (rarely - less than 3 times per month, sometimes - less than 3 times a week but more than 3 per month, regularly - 3 or more times each week), education (degree level, post-16 but below degree, to age 16 or unspecified), socio-economic status (tertiles), height (sex-specific tertiles), BMI (sex-specific tertiles), and for women we also adjusted for: HRT use ever (yes, no), and one or more children (yes,no). Baseline was taken as: never smoker, rarely drink, brisk walking pace, degree-level education, minimum deprivation tertile, minimum height tertile in men (or women), middle BMI tertile in men (or women), and women with no children or HRT use. Only diseases with at least 140 cases were considered. This ensured there were at least 10 cases per parameter to adjust from baseline, even if a parametric e.g. Weibull model with an extra two parameters to fit the baseline hazard function were considered [12]. Sensitivity analyses excluded participants with a broader range of prior diseases, leading to fewer total cases and fewer diseases included in the study. Analyses were multiply adjusted. There were less than $1 \%$ missing values, allowing a complete case analysis. Numerical work and plots used R [15], and packages used here included: survival[16] and grr[17].

Attribution fractions for the UK Biobank population were considered for three situations: observed population verus all smoking status as never-smoked, observed population versus middle BMI tertile, and observed population versus neversmoked and middle BMI tertile. The latter case is comparing the correlated exposures of BMI and smoking status in the observed population, to a situation where BMI and smoking are set to their baseline values. Because the baseline BMI tertile is the middle tertile, current smokers could be correlated with either the top or bottom BMI tertile. Frequency of alcohol consumption was adjusted for but not studied, because it is a less precise measure than smoking status or BMI, and it is known to have inconsistent associations with disease risk in different studies.

\section{Results}

Plots and tables only included diseases with statistically significant associations for current smoking versus never smoked or maximum versus middle BMI tertile, after an FDR multiple-testing adjustment. Where results involve both smoking and BMI then diseases were included if they are included in either of the smoking-only, or BMI-only results. This left 129 diseases associated with BMI, 153 diseases associated with smoking, and 226 diseases that were associated with either smoking or BMI. To explore the sensitivity of the estimates to the strength of confounding factors, estimates made using Eq. 19 and 18 were compared (figure 5 in the Appendix). As expected, the influences of confounding are more noticeable for smaller attributable fractions, but even in those cases, the estimates rarely differ by more than about $20 \%$. With a handful of exceptions, such as Parkinson's disease (G20), estimates with Eq. 19 were larger than with 18, as would be expected if the influence of smoking and BMI were positively correlated with the influence of the confounding factors in the model.

Figure 3 shows the median attributable fractions for the combined influence of smoking and BMI on the incidence of disease in each ICD-10 chapter, with the width of the bar plots proportional to the number of diseases included in the estimate. Diseases of the respiratory system (X) have the largest median attribution fraction, of about 0.3 , closely followed by endocrine, nutritional, and metabolic diseases, that are both almost double the next largest values. Diseases of the skin and subcutaneous tissues (XII) and of the nervous system (VI), both have median attribution fractions near 0.15. Neoplasms 
medRxiv preprint doi: https://doi.org/10.1101/2021.12.24.21268368; this version posted December 25, 2021. The copyright holder for this preprint (which was not certified by peer review) is the author/funder, who has granted medRxiv a license to display the preprint in

All rights reserved. No reuse allowed without permission.

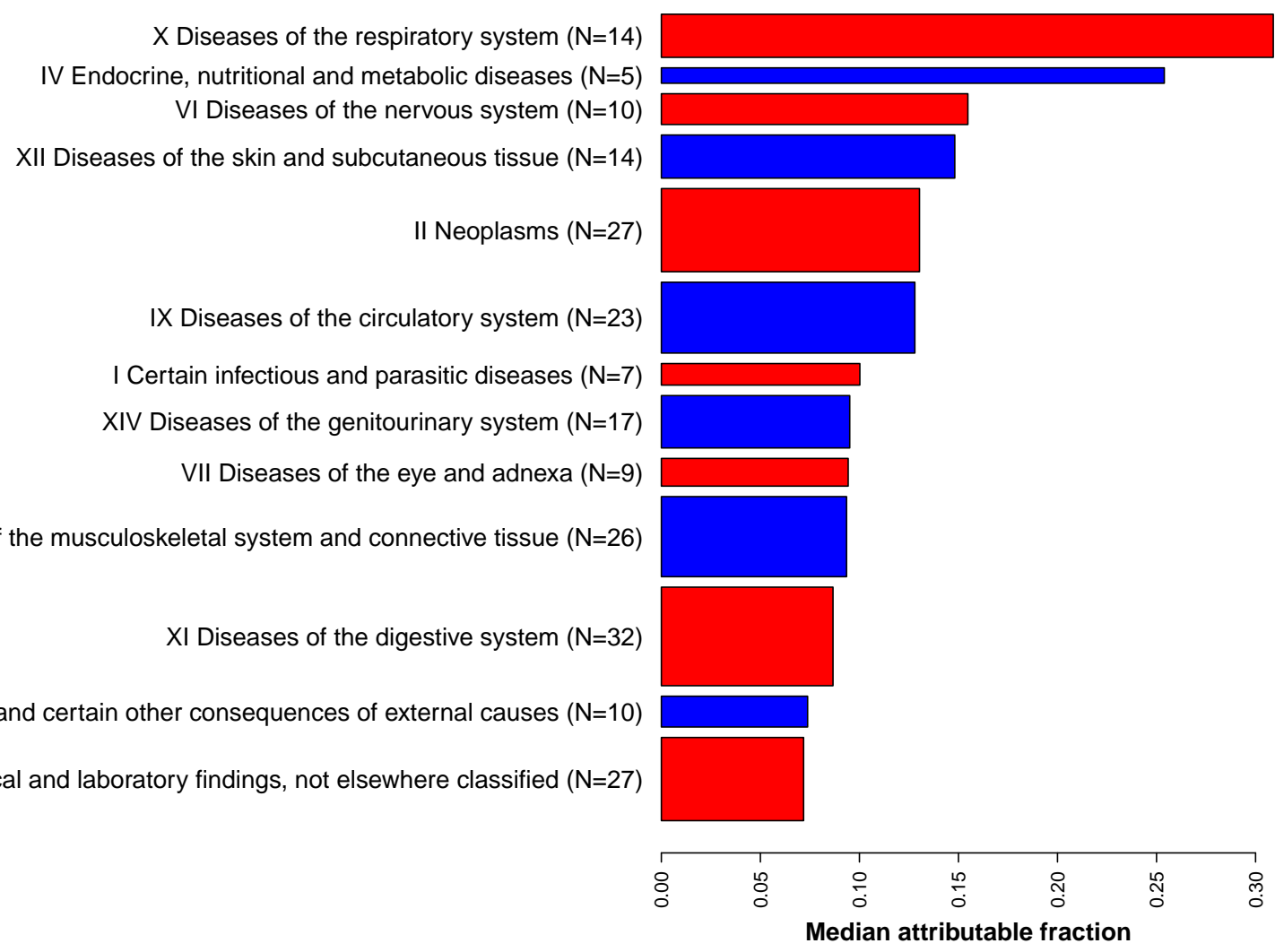

Figure 3: Median attributable fractions for each ICD-10 chapter with at least 5 diseases where $A_{f}>0.2$. Bar widths are proportional to the number of diseases in each chapter.

and circulatory diseases account for $22 \%$ of all the diseases, and have the next largest median attributable fractions. There are seven chapters with median attribution fractions greater than 0.1 , and these include 100 diseases, 50 of which are neoplasms and diseases of the circulatory system.

The $26 \%$ of diseases that had $A_{f} \geq 0.2$ are listed in table 1 . There are 11 diseases with $A_{f} \geq 0.5$ and 21 with $A_{f} \geq 0.35$. Given the limitations of the analysis and the potential for regression dilution bias, it is possible that more than 11 diseases could have $A_{f} \geq 0.5$. For diseases with more than half the cases attributed to smoking and BMI, it seems reasonable to regard smoking and BMI as "pathogenic", in a similar way that strong genetic risk factors are often described as pathogenic. One third of the 226 diseases had an attributable fraction with $\left|A_{f}\right|>0.17$, and two thirds had $\left|A_{f}\right|>0.06$. Although the mean attribution fraction for the combined influence of smoking and BMI was $\simeq 15 \%$, the estimated attributable number of extra cases was only $\simeq 8 \%$, reflecting the fact that the most common diseases (with the most cases), tended to have smaller attributable fractions.

Diseases were ranked in terms of their attribution fractions for smoking and BMI (figure 4). Figure 4 identifies an important point, that even established risk factors such as smoking and BMI can have protective associations with some diseases. The 20 diseases that smoking and BMI have the strongest protective associations with are listed in table 2 . There were 12 diseases whose protective association had an attributable fraction with magnitude greater than 0.1 , and 3 with magnitude greater than 0.2. Melanoma in situ (D03), had the strongest protective association of -0.29 , where the sign is taken to indicate the direction of effect as discussed in "Number of attributed cases".

\section{Sensitivity analysis}

Participants with hospital reports of prior cancers other than non-melanoma skin cancers were excluded from the main study, but self-reported cancers or other prior diseases were not. It is possible for example, that a heart attack might be followed by weight loss, and including participants with prior heart attacks could weaken a potential association between BMI and 
medRxiv preprint doi: https://doi.org/10.1101/2021.12.24.21268368; this version posted December 25, 2021. The copyright holder for this preprint (which was not certified by peer review) is the author/funder, who has granted medRxiv a license to display the preprint in

perpetuity.

All rights reserved. No reuse allowed without permission.

\begin{tabular}{|c|c|c|c|c|c|c|}
\hline Disease & Sex & $\mathrm{N}$ & $N_{A_{f}}$ & Rank & $A_{f}$ & Rank $A_{f}$ \\
\hline E66 Obesity & $\mathrm{F}$ & 311 & 306 & 24 & 0.98 & 1 \\
\hline J44.1 Chronic obstructive pulmonary disease with acute exacerbation, unspecified & $\mathrm{F}$ & 209 & 193 & 53 & 0.92 & 2 \\
\hline J44.0 Chronic obstructive pulmonary disease with acute lower respiratory infection & $\mathrm{F}$ & 416 & 376 & 17 & 0.90 & 3 \\
\hline J44.0 Chronic obstructive pulmonary disease with acute lower respiratory infection & M & 417 & 374 & 18 & 0.90 & 4 \\
\hline J44.1 Chronic obstructive pulmonary disease with acute exacerbation, unspecified & M & 261 & 230 & 41 & 0.88 & 5 \\
\hline C34 Malignant neoplasm of bronchus and lung & M & 1018 & 833 & 4 & 0.82 & 6 \\
\hline I70 Atherosclerosis & M & 156 & 114 & 86 & 0.73 & 7 \\
\hline C34 Malignant neoplasm of bronchus and lung & $\mathrm{F}$ & 996 & 710 & 6 & 0.71 & 8 \\
\hline E11 Non-insulin-dependent diabetes mellitus & M & 206 & 114 & 86 & 0.55 & 9 \\
\hline I71 Aortic aneurysm and dissection & M & 402 & 222 & 44 & 0.55 & 10 \\
\hline R04.2 Haemoptysis & M & 314 & 162 & 60 & 0.52 & 11 \\
\hline C15 Malignant neoplasm of oesophagus & M & 473 & 220 & 46 & 0.47 & 12 \\
\hline R91 Abnormal findings on diagnostic imaging of lung & M & 358 & 162 & 60 & 0.45 & 13 \\
\hline C67 Malignant neoplasm of bladder & M & 1063 & 438 & 9 & 0.41 & 14 \\
\hline R91 Abnormal findings on diagnostic imaging of lung & $\mathrm{F}$ & 372 & 151 & 65 & 0.41 & 15 \\
\hline I50 Heart failure & $\mathrm{F}$ & 280 & 111 & 88 & 0.40 & 16 \\
\hline K42 Umbilical hernia & $\mathrm{F}$ & 297 & 111 & 88 & 0.37 & 17 \\
\hline G47.3 Sleep apnoea & $\mathrm{F}$ & 381 & 139 & 69 & 0.36 & 18 \\
\hline J84 Other interstitial pulmonary diseases & $\mathrm{F}$ & 177 & 63 & 117 & 0.35 & 19 \\
\hline I50 Heart failure & M & 359 & 125 & 75 & 0.35 & 20 \\
\hline R04.2 Haemoptysis & $\mathrm{F}$ & 239 & 83 & 103 & 0.35 & 21 \\
\hline J10 Influenza due to identified influenza virus & $\mathrm{F}$ & 211 & 69 & 114 & 0.33 & 22 \\
\hline M13 Other arthritis & M & 215 & 70 & 112 & 0.32 & 23 \\
\hline R29.6 Tendency to fall, not elsewhere classified & M & 182 & 58 & 123 & 0.32 & 24 \\
\hline A41 Other septicaemia & $\mathrm{F}$ & 957 & 303 & 25 & 0.32 & 25 \\
\hline J18 Pneumonia, organism unspecified & M & 3011 & 944 & 2 & 0.31 & 26 \\
\hline C22 Malignant neoplasm of liver and intrahepatic bile ducts & M & 171 & 54 & 126 & 0.31 & 27 \\
\hline L72.0 Epidermal cyst & M & 456 & 139 & 69 & 0.30 & 28 \\
\hline J18 Pneumonia, organism unspecified & $\mathrm{F}$ & 2777 & 845 & 3 & 0.30 & 29 \\
\hline G47.3 Sleep apnoea & M & 776 & 236 & 39 & 0.30 & 30 \\
\hline N17 Acute renal failure & $\mathrm{F}$ & 333 & 99 & 94 & 0.30 & 31 \\
\hline G44.2 Tension-type headache & $\mathrm{F}$ & 152 & 44 & 135 & 0.29 & 32 \\
\hline K43 Ventral hernia & $\mathrm{F}$ & 377 & 108 & 90 & 0.29 & 33 \\
\hline G62 Other polyneuropathies & M & 175 & 50 & 130 & 0.29 & 34 \\
\hline C16 Malignant neoplasm of stomach & M & 260 & 70 & 112 & 0.27 & 35 \\
\hline B37 Candidiasis & M & 173 & 47 & 132 & 0.27 & 36 \\
\hline R06.0 Dyspnoea & M & 650 & 176 & 58 & 0.27 & 37 \\
\hline I26 Pulmonary embolism & $\mathrm{F}$ & 836 & 225 & 43 & 0.27 & 38 \\
\hline R63.4 Abnormal weight loss & $\mathrm{F}$ & 485 & 125 & 75 & 0.26 & 39 \\
\hline J84 Other interstitial pulmonary diseases & M & 234 & 60 & 119 & 0.26 & 40 \\
\hline J22 Unspecified acute lower respiratory infection & $\mathrm{F}$ & 1506 & 386 & 15 & 0.26 & 41 \\
\hline E87.1 Hypo-osmolality and hyponatraemia & M & 234 & 59 & 120 & 0.25 & 42 \\
\hline I25.9 Chronic ischaemic heart disease, unspecified & M & 254 & 63 & 117 & 0.25 & 43 \\
\hline M48 Other spondylopathies & $\mathrm{F}$ & 573 & 139 & 69 & 0.24 & 44 \\
\hline K62.1 Rectal polyp & M & 1143 & 275 & 32 & 0.24 & 45 \\
\hline N17 Acute renal failure & M & 562 & 135 & 71 & 0.24 & 46 \\
\hline H02.4 Ptosis of eyelid & M & 253 & 59 & 120 & 0.23 & 47 \\
\hline C90 Multiple myeloma and malignant plasma cell neoplasms & $\mathrm{F}$ & 247 & 58 & 123 & 0.23 & 48 \\
\hline M47 Spondylosis & M & 338 & 79 & 105 & 0.23 & 49 \\
\hline L60 Nail disorders & $\mathrm{F}$ & 236 & 55 & 125 & 0.23 & 50 \\
\hline R13 Dysphagia & M & 959 & 218 & 47 & 0.23 & 51 \\
\hline J90 Pleural effusion, not elsewhere classified & M & 524 & 119 & 82 & 0.23 & 52 \\
\hline J22 Unspecified acute lower respiratory infection & M & 1349 & 300 & 27 & 0.22 & 53 \\
\hline L03 Cellulitis & M & 2036 & 450 & 8 & 0.22 & 54 \\
\hline M81 Osteoporosis without pathological fracture & $\mathrm{F}$ & 946 & 204 & 50 & 0.22 & 55 \\
\hline K92.0 Haematemesis & M & 156 & 33 & 152 & 0.21 & 56 \\
\hline L03 Cellulitis & $\mathrm{F}$ & 1602 & 333 & 21 & 0.21 & 57 \\
\hline K25 Gastric ulcer & M & 338 & 70 & 112 & 0.21 & 58 \\
\hline I64 Stroke, not specified as haemorrhage or infarction & M & 173 & 35 & 148 & 0.20 & 59 \\
\hline
\end{tabular}

Table 1: Attributable fractions $A_{f}$ for both smoking and BMI are estimated with Eq. 19, ranked, and listed if $A_{f} \geq 0.2$. Colours: $A_{f} \geq 0.5$ (red), $0.5>A_{f} \geq 0.35$ (orange), $0.35>A_{f} \geq 0.2$ (yellow). Sex: diseases in males (M) or females (F), $N$ : total cases, $N_{A_{f}}$ : attributed cases. 
Smoking

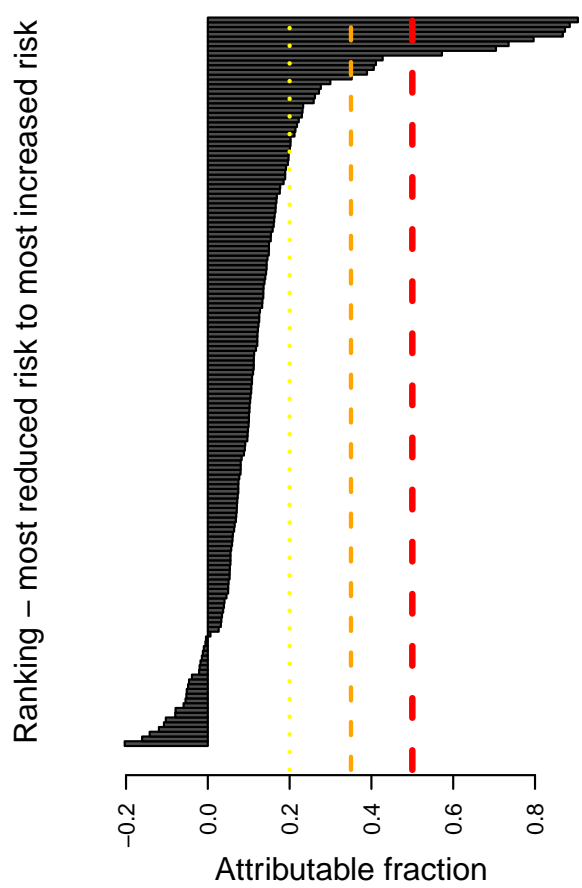

BMI

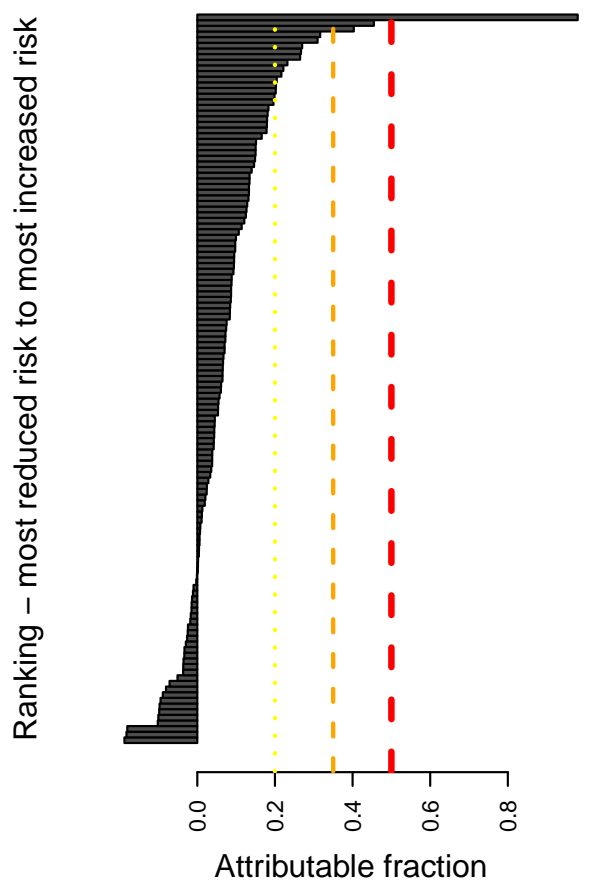

Smoking and BMI

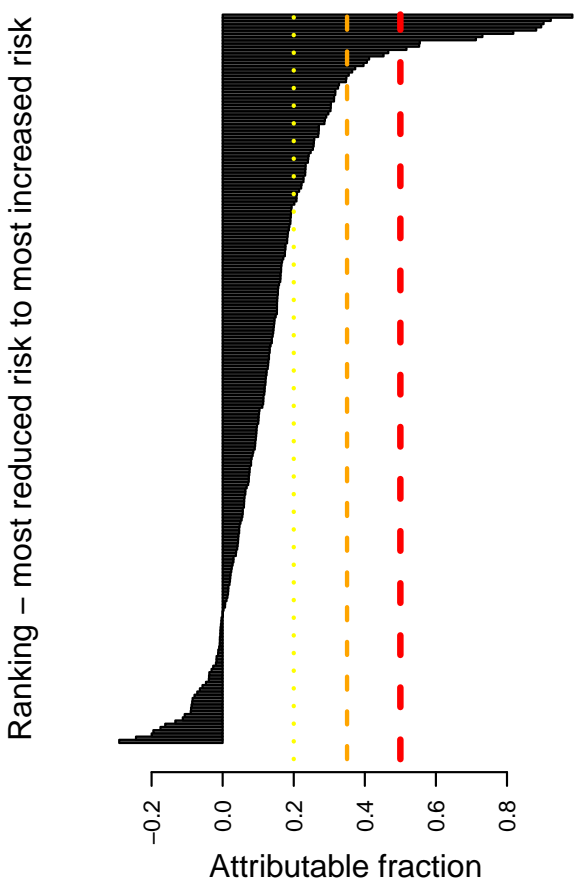

Figure 4: For diseases with a statistically significant association with smoking or BMI after an FDR multiple-testing adjustment, attributable fractions $A_{f}$ were calculated with Eq. 19. Lines indicate $A_{f}=0.5$ (red), $A_{f}=0.35$ (orange), $A_{f}=0.2$ (yellow). $A_{f}<0$ indicates a protective association.

\begin{tabular}{|c|c|c|c|c|c|c|}
\hline Disease & Sex & $\mathrm{N}$ & $N_{A_{f}}$ & Rank & $A_{f}$ & Rank $A_{f}$ \\
\hline D03 Melanoma in situ & M & 272 & -79 & 14 & -0.290 & 1 \\
\hline K31.7 Polyp of stomach and duodenum & $\mathrm{F}$ & 870 & -212 & 9 & -0.240 & 2 \\
\hline N41 Inflammatory diseases of prostate & $\mathrm{M}$ & 572 & -114 & 10 & -0.200 & 3 \\
\hline N81 Female genital prolapse & $\mathrm{F}$ & 4199 & -819 & 1 & -0.190 & 4 \\
\hline R79 Other abnormal findings of blood chemistry & M & 1905 & -333 & 5 & -0.170 & 5 \\
\hline S02 Fracture of skull and facial bones & M & 355 & -57 & 18 & -0.160 & 6 \\
\hline C43 Malignant melanoma of skin & M & 723 & -96 & 11 & -0.130 & 7 \\
\hline S76.1 Injury of quadriceps muscle and tendon & M & 215 & -24 & 25 & -0.110 & 8 \\
\hline M20.1 Hallux valgus (acquired) & $\mathrm{F}$ & 2875 & -307 & 6 & -0.110 & 9 \\
\hline C61 Malignant neoplasm of prostate & M & 5800 & -521 & 2 & -0.090 & 10 \\
\hline B34 Viral infection of unspecified site & M & 319 & -28 & 23 & -0.089 & 11 \\
\hline N40 Hyperplasia of prostate & M & 3928 & -344 & 4 & -0.088 & 12 \\
\hline M16 Coxarthrosis [arthrosis of hip] & M & 3167 & -272 & 7 & -0.086 & 13 \\
\hline J90 Pleural effusion, not elsewhere classified & $\mathrm{F}$ & 327 & -28 & 23 & -0.084 & 14 \\
\hline K40 Inguinal hernia & $\mathrm{F}$ & 493 & -39 & 20 & -0.079 & 15 \\
\hline R19.8 Other specified symptoms and signs involving the digestive system and abdomen & $\mathrm{F}$ & 302 & -22 & 27 & -0.072 & 16 \\
\hline C44 Other malignant neoplasms of skin & M & 6095 & -391 & 3 & -0.064 & 17 \\
\hline K31.7 Polyp of stomach and duodenum & M & 300 & -17 & 31 & -0.057 & 18 \\
\hline
\end{tabular}

Table 2: Diseases with the strongest protective associations, ranked by the proportion of disease attributed to a combination of smoking and BMI $\left(A_{f}\right)$. Sex indicates diseases in males (M) or females $(\mathrm{F}), N$ are total cases, $N_{A_{f}}$ are the number of cases attributed to smoking and BMI, $A_{f}$ is the attributable fraction.

heart disease. In contrast, smoking might increase the risk of some diseases for which a substantial proportion occur before entry into the UK Biobank study. In that case, including participants with the prior disease might strengthen the associations. The question of how best to study sequences of disease is an example where causal understanding is not enough, and new statistical methods or data are likely to be required. It might be an intractable question, due to the vast possible combinations 
medRxiv preprint doi: https://doi.org/10.1101/2021.12.24.21268368; this version posted December 25, 2021. The copyright holder for this preprint (which was not certified by peer review) is the author/funder, who has granted medRxiv a license to display the preprint in

All rights reserved. No reuse allowed without permission.

\begin{tabular}{llrrrrr}
\hline \multicolumn{1}{c}{ Disease } & $\mathrm{Sex}$ & $\mathrm{N}$ & $N_{A_{f}}$ & Rank & $A_{f}$ & Rank $A_{f}$ \\
\hline S00.8 Superficial injury of other parts of head & $\mathrm{F}$ & 171 & 39 & 135 & 0.23 & 44 \\
S92 Fracture of foot, except ankle & $\mathrm{M}$ & 168 & 38 & 138 & 0.22 & 46 \\
E21 Hyperparathyroidism and other disorders of parathyroid gland & $\mathrm{F}$ & 296 & 66 & 106 & 0.22 & 48 \\
I21 Acute myocardial infarction & $\mathrm{F}$ & 1125 & 243 & 28 & 0.22 & 50 \\
M79.6 Pain in limb & $\mathrm{F}$ & 920 & 198 & 41 & 0.22 & 51 \\
M17 Gonarthrosis [arthrosis of knee] & $\mathrm{F}$ & 3623 & 735 & 3 & 0.20 & 57 \\
\hline
\end{tabular}

Table 3: The sensitivity analyses found six additional diseases with $A_{f} \geq 0.2$, for the combination of both smoking and BMI, that would have appeared in table 1. Sex: diseases in males (M) or females (F), $N$ : total cases, $N_{A_{f}}$ : attributed cases.

of sequences of 100s of diseases, and it is further complicated by the complex time-dependent exposures and accumulation of genetic mutations that any individual experiences. Therefore a senstivity analysis compared the paper's main results with a second analysis that excluded participants who had reported any cancer other than non-melanoma skin cancer, or any serious cardiovascular disease of heart disease, stroke, arterial or pulmonary embolisms, or subarachnoid haemorrhage.

Differences between the main study and the sensitivity analysis were small (for full details see the Supplementary Material). The difference in attribution fractions between the two studies had a mean and median of -0.006 and -0.005 respectively, and a standard deviation of 0.029 . The differences in magnitude were typically equivalent to about $10 \%$. The attribution fractions of six diseases changed by more than 0.05 . These included increased attributable fractions for: I50 - heart failure in women (0.40 to 0.48$), \mathrm{R} 29.6$ - tendency to fall in men ( 0.32 to 0.41$)$, and decreases in: C16 - stomach cancer in men $(0.27$ to 0.21$), \mathrm{J} 10$ - influenza in women (0.33 to 0.27$)$, J22 - lower respiratory infections in men (0.24 to 0.17$)$, and N17 - acute renal failure (0.24 to 0.17). Overall the differences were small, but larger than the approximations in Eq. 19 would cause.

\section{Discussion}

\section{Attribution fractions}

Similar attribution formulae to that used here have existed in published literature since at least 1998 [4]. A contribution of this paper is to recognise that for a particular causal model such as that in figure 1, the attribution fractions can only be used with a restricted range of potential risk modifiers, whose associations have a causal interpretation. The consideration of attributable fractions from the perspective of causal inference, has helped to highlight several important points:

1. It is not possible to estimate causal associations and attributable fractions for all factors that are thought to be associated with disease risk.

For example, if the causal model is not known sufficiently well, then it may not be possible to correctly adjust for confounding. Alternately, if the measurement is too imprecise e.g. socio-economic status is likely to capture the influence of several factors that may include exposure to pollution, poor quality diet, poor living and working conditions, etc, then it may not be possible to estimate a meaningful causal association - for example, someone with an equivalent socio-economic status in a different country would experience different exposures and have different causal factors that influence their health.

2. Estimates of causal associations cannot always be obtained directly from a single multiply-adjusted analysis, and may need several different analyses of the same dataset, as required by the causal model.

For example, changes in systolic blood pressure (SBP) can be caused by smoking or BMI, and therefore SBP should not be adjusted for if we are interested in the influence of smoking and BMI on disease risk. In contrast, if our interest was in SBP, then we would need to adjust for BMI and smoking if they can modify disease risk in any way other than through changes in SBP. 
medRxiv preprint doi: https://doi.org/10.1101/2021.12.24.21268368; this version posted December 25 , 2021. The copyright holder for this preprint (which was not certified by peer review) is the author/funder, who has granted medRxiv a license to display the preprint in

All rights reserved. No reuse allowed without permission.

\section{Attribution to smoking and BMI}

The original intention of this work was to characterise diseases through the overall modifiability of disease risk by established risk factors. To do so as rigorously as possible, a causal model similar to figure 1 was assumed, and the strongest established risk factors of smoking and BMI were considered. Under the model in figure 1, Eq. 19 is intended to provide attribution fractions for the causal increase in disease incidence due to smoking and BMI, while accounting for confounding factors that would also be expected to modify disease incidence. The study considered the most common diseases in UK Biobank, and highlighted two important points:

1. Attribution fractions for smoking, and BMI, are very heterogeneous, and can even involve a reduction in risk (table 2).

2. Some associations are extremely strong, for example with $A_{f}>0.5$.

The first point highlights a difficulty in optimising lifestyle and drug treatments - changes in lifestyle or medication are likely to have a heterogeneous influence on disease risk, with some risks lowered but others potentially increased. In the same way that some germline genetic risk factors are described as pathogenic when they substantially increase your risk of disease, it seems reasonable to describe the influence of smoking and BMI as "pathogenic", for diseases with high attributable fractions such as $A_{f}>0.5$. Based on the estimates here, eliminating smoking and controlling BMI would be expected to prevent the majority of those diseases in an equivalent population.

The attribution fractions allow us to identify the diseases for which a change in lifestyle is likely to have the greatest impact. From a population perspective, eliminating smoking and controlling BMI in an equivalent population would be expected to avoid: the majority of diseases with $A_{f}>0.5$ (red in table 1), between one third and one half of diseases with $0.35>A_{f}>0.5$ (orange in table 1), between one fifth and one third of diseases with $0.2>A_{f}>0.35$ (yellow in table 1). This slightly ad-hoc categorisation of diseases by their attributable fractions provides an approximate indication of how the patterns of disease would be expected to change if smoking were eliminated and BMI were controlled in a population that was otherwise similar to that in UK Biobank.

The attributed fractions would be larger if more of the population were exposed. For examples where a binary exposure $\mathrm{X}$ is uncorrelated with $\mathrm{W}$ or confounders $\mathrm{Z}$, then Appendix $\mathrm{C}$ shows that provided $p(R-1) \ll 1$, where $R$ is the relative risk and $p$ is the proportion of the population that are exposed, then $A_{f} \simeq p(R-1)$. In that case, if the exposed proportion $p$ were halved, then so would the attributable fraction. This highlights a limitation of Eqs. 16, 19, and 17 - they measure the proportion of disease in a population that is attributed to an exposure, but a clinician might be more interested in what proportion of disease in smokers is attributable to smoking, and an individual might be more interested in whether their risk of serious disease or death is substantially changed. Such questions that refer to individuals as opposed to populations might best be tackled with counterfactual arguments and measurements such as Eq. 28. An alternative approach is to consider the "probability of necessity" [6,7], that in principle allows the assessment of whether it is more probable than not that the disease would not have occurred if you had not been exposed to e.g. smoking. Such approaches allow specific individual cases to be assessed, but do not provide an overall characterisation of an exposure's influence on population health.

When considering the attribution fraction for both smoking and BMI together, the study included diseases with statistically significant associations with either smoking or BMI. In this situation, especially when the number of cases are few, the estimates for one of the two parameters can in principle be both large and imprecise. This has the potential to produce misleading estimates for the joint attribution fraction of both smoking and BMI. A notable example is the strong protective association of smoking with Parkinson's disease (see table 7), that was substantially weakened in table 2 by the association with BMI, even though the association with BMI was not statistically significant. This appears to be an isolated example, and the potential problem will reduce with more cases, but it highlights the importance of also considering the attribution fractions associated with each separate exposure.

\section{Meta analyses}

If estimates are to be used in a meta-analyses of data that have not originated from a randomised control trial, then it is essential to ensure that estimates are of causal associations. It is possible that some reported estimates from observational studies will measure the causal influence of a potential risk factor, such as BMI, alcohol, and smoking in the first example 
medRxiv preprint doi: https://doi.org/10.1101/2021.12.24.21268368; this version posted December 25 , 2021. The copyright holder for this preprint (which was not certified by peer review) is the author/funder, who has granted medRxiv a license to display the preprint in

All rights reserved. No reuse allowed without permission.

considered here, but this is unlikely to be true for all variables that are adjusted for. If a study has inappropriately adjusted for potential confounding variables, then the data cannot be included in the meta-analysis. The assessment of this requires a good causal understanding of how the risk factor of interest modifies disease risk, and the potential confounding factors that need adjusting for. Disagreement between studies may indicate incomplete understanding of the underlying causal model, with inappropriate or insufficient adjustment for confounding factors. In the common situation where uncertainty of the causal processes linking exposure $X$ to disease risk remain, then the standard methods and cautious reporting of conventional epidemiology must remain $[2,8]$.

\section{Conclusions}

The aim was to characterise how the risk of common diseases are modified by established risk factors, and to identify which disease risks are the most sensitive to them. This requires estimates of causal associations between potential risk factors and disease risk, and it was shown that many conventional epidemiological studies can provide these. This requires a sufficiently good model of the causal processes, and appropriate adjustment for confounders. This limits the range of potential risk factors that can be studied, but will often include important risk factors such as smoking and BMI.

The first generic example was a causal model with risk factors $X$ that satisfied the "backdoor criteria" [7], a situation that is likely to commonly occur. The second example considered the "frontdoor" criteria, that can allow causal estimations in the presence of unmeasured confounders, when the influence of $X$ is mediated by a measurable variable $Z$. In this case, for the simple example considered (with rare disease incidence, and the linear influence of a continuous exposure mediated by a normally distributed continuous variable whose mean is linearly related to the exposure), the causal estimate is the same as you get from a mediation analysis with measured confounders. With hindsight, this might have been anticipated by observing that for this situation in the limit of rare diseases studied with a proportional hazards model, the confounding terms do not appear in the results of mediation studies for natural direct, and indirect, effects [9].

The causal attribution of exposures to population disease risk was considered for the situation corresponding to figure 1, leading to equations 16 and 19. These are similar to the expression used by the World Health Organisation, but accounts for correlations between exposures and confounding factors. The attribution fractions $\left(A_{f}\right)$ allowed a simple categorisation of diseases to indicate how the patterns of disease might change if smoking were eliminated and BMI controlled, in an equivalent population to UK Biobank. For the combined influence of smoking and BMI compared to baseline of never smoking and mid-tertile BMI, this identified 11 diseases with $A_{f}>0.5$, a further 10 with $0.35<A_{f}<0.5$, and a total of 59 with $A_{f}>0.2$. Similar results were found by a sensitivity analysis. Because a large proportion of the population studied were "unexposed" (non-smoking and a healthy weight), the attribution fraction for e.g. disease in the population attributed to smoking, will often be less than that for an individual. Attribution fractions for individuals are simpler expressions (Eq. 28), because they do not need to account for complex correlations that occur between risk factors in a population.

In summary, this article has attempted to link established epidemiological methods $[2,3]$ with newer techniques in causal inference [6,7], to help clarify the definition of attribution fractions. This subsequently allowed a quantitative characterisation of the causal associations between smoking, BMI, and the incidence of disease in the UK Biobank cohort [10], using their attribution fractions $\left(A_{f}\right)$, and categorising them into groups with similar $A_{f}$. These help to indicate the expected change in patterns of disease due to changes e.g. to a healthier lifestyle, for an equivalent population.

\section{Data availability}

UK Biobank data can be accessed by application through www.ukbiobank.ac.uk, and summary data produced during this study will become available from:

https://osf.io/

UK Biobank has approval by the Research Ethics Committee (REC) under approval number 16/NW/0274. UK Biobank obtained participant's consent for the data to be used for health-related research.

\section{Code availability}

$\mathrm{R}$ code used to produce figures from summary data will become available with the summary data at: 
medRxiv preprint doi: https://doi.org/10.1101/2021.12.24.21268368; this version posted December 25,2021 . The copyright holder for this preprint (which was not certified by peer review) is the author/funder, who has granted medRxiv a license to display the preprint in

All rights reserved. No reuse allowed without permission.

https://osf.io/

The full code for use with non-summary data will be returned with other results to UK Biobank (see www.ukbiobank.ac.uk).

\section{Acknowledgements}

Thank you to Professor Robert Clarke for suggesting a sensitivity analysis to strengthen the results. This research has been conducted using the UK Biobank resource under application number 42583. Anthony Webster is supported by an intermediate research fellowship from the Nuffield Department of Population Health (NDPH), University of Oxford.

\section{Competing interests}

The author declares no competing interests.

\section{References}

[1] World Health Organization, Global health risks: mortality and burden of disease attributable to selected major risks, World Health Organization (2009).

[2] T.L. Lash, T.J. VanderWeele, S. Haneuse, K.J. Rothman, Modern Epidemiology, Fourth Edition, Wolters Kluwer, (2021).

[3] D. Collett Modelling Survival Data in Medical Research, New York: Chapman and Hall/CRC, 3rd edition, (2014).

[4] Rockhill, B. Newman, B. and Weinberg, C. Use and misuse of population attributable fractions American Journal of Public Health, 88, 15-19, (1998).

[5] M.A. Mansournia, A. Douglas G, Population attributable fraction, BMJ - British Medical Journal, 360, k757, (2018).

[6] J. Pearl Causality, 2nd ed., John Wilely \& Sons Ltd, (2009).

[7] J. Pearl, M. Glymour, N.P. Jewell, Causal Inference In Statistics, Cambridge University Press, (2016).

[8] Shimonovich, M., Pearce, A., Thomson, H. et al. Assessing causality in epidemiology: revisiting Bradford Hill to incorporate developments in causal thinking Eur. J. Epidemiol. 36, 873-887 (2021).

[9] T.J. VanderWeele Explanation in Causal Inference, Oxford University Press, (2015).

[10] C. Bycroft et al. The UK Biobank resource with deep phenotyping and genomic data Nature 562, 203-209, (2018).

[11] E.T. Jaynes Probability Theory: The Logic of Science, Cambridge University Press, (2003).

[12] A.J. Webster, R. Clarke Sporadic, late-onset, and multistage diseases, medRxiv 2021.12.15.21267843, Cold Spring Harbor Laboratory Press, (2021).

[13] Webster, A.J., Gaitskell, K., Turnbull, I., Cairns B.J., Clarke R. Characterisation, identification, clustering, and classification of disease Scientific Reports 11, 5405 (2021).

[14] World Health Organisation, International Statistical Classification of Diseases (ICD), www.who.int/standards/classifications/classification-of-diseases, (2021).

[15] R Core Team. R: A Language and Environment for Statistical Computing R Foundation for Statistical Computing, Vienna, Austria, www.R-project.org, (2020).

[16] Therneau, T.M. A Package for Survival Analysis in R, CRAN.R-project.org/package=survival, (2021).

[17] Varrichio, C. grr: Alternative Implementations of Base R Functions, CRAN.R-project.org/package=grr, (2016).

[18] Yang, L. Kartsonaki, C, Yao, P. The relative and attributable risks of cardia and non-cardia gastric cancer associated with Helicobacter pylori infection in China: a case-cohort study, The Lancet Public Health, 6, Issue 12 , e888 - e896. 


\section{A Probability densities and hazard functions}

Using Eq. 4 we can calculate the probability density function,

$$
\begin{aligned}
P(Y=1, T \in(t, t+\delta t) \mid \operatorname{do}(X=x)) & =\delta t \frac{d}{d t} P(Y=1, T<t \mid \operatorname{do}(X=x)) \\
& \simeq e^{\eta_{x}} A_{Z} h_{0}(t) \delta t \\
& =e^{\eta_{x}} A_{Z} P\left(Y=1, T \in(t, t+\delta t) \mid X=x_{0}, Z=z_{0}\right)
\end{aligned}
$$

This allows a hazard function (usually defined as $h=f / S=f /(1-F)$ ), to be defined as,

$$
h(t \mid \operatorname{do}(X=x)) \delta t=\frac{P(Y=1, T \in(t, t+\delta t) \mid \operatorname{do}(X))}{1-P(Y=1, T<t \mid \operatorname{do}(X))} \simeq e^{\eta_{x}} A_{Z} h_{0}(t) \delta t
$$

Alternately, we could have argued that most diseases in UK Biobank are sufficiently rare that we can approximate $f(t) \simeq$ $h(t)$, and hence that $h(t \mid \operatorname{do}(X=x)) \delta t \simeq P(Y=1, T \in(t, t+\delta t) \mid \operatorname{do}(X=x))$ Therefore for combinations of confounders and risk factors that satisfy the "backdoor criteria" [7] (such as those in figure 1), and disease incidence that is sufficiently rare (which includes most studies of the first incidence of a disease in UK Biobank data [12]),

$$
\frac{h(t \mid \operatorname{do}(X=x))}{h\left(t \mid \operatorname{do}\left(X=x_{0}\right)\right)} \simeq e^{\eta_{x}}
$$

This indicates that if we are interested in the relative difference in disease rates that would be caused by changing from the baseline value $X=x_{0}$, to $X=x$ (e.g. to reduce risk), then the result is given in terms of the usual relative risk, but without terms in $Z$ (corresponding to $Z=z_{0}$ ). This indicates that causal inferences for $x$ using the relative risk, will be correct in these circumstances. Any confounders $Z \neq z_{0}$ that may appear in an estimated relative risk, are set at their baseline values $Z=z_{0}$ to estimate Eqs. 31 and 6.

Note that hazard functions do not give a probability in the conventional sense, and are not normalised to 1 for example. To ensure the above calculations were done correctly and will e.g. normalise to 1 (within the limits of the approximations made), it was essential for the arguments to have used probabilities (for which the backdoor theorem applies). For a clear exposition of when a particular situation will satisfy the "backdoor criterion", please refer to the references [6, 7].

\section{B Bounds on attribution fractions}

By definition, if the relative risks $e^{\eta_{x}}$ and $e^{\eta_{w}+\eta_{z}}$ are positively correlated, then $E\left[e^{\eta_{x}} e^{\eta_{w}+\eta_{z}}\right]-E\left[e^{\eta_{x}}\right] E\left[e^{\eta_{w}+\eta_{z}}\right] \geq 0$, where $E$ is used to denote expectations. Therefore, using $\exp (s) \geq 0$ for any real-valued $s$, we can rearrange the inequality as,

$$
\begin{array}{ll}
E\left[e^{\eta_{x}} e^{\eta_{w}+\eta_{z}}\right] & \geq E\left[e^{\eta_{x}}\right] E\left[e^{\eta_{w}+\eta_{z}}\right] \\
\frac{1}{E\left[e^{\eta_{x}}\right]} & \geq \frac{E\left[e^{\eta_{w}+\eta_{z}}\right]}{E\left[e^{\eta_{x}} e^{\eta_{w}+\eta_{z}}\right]} \\
1-\frac{\left.E e^{\eta_{w}+\eta_{z}}\right]}{E\left[e^{\eta_{x}} e^{\eta_{w}+\eta_{z}}\right]} & \geq 1-\frac{1}{E\left[e^{\eta_{x}}\right]}
\end{array}
$$

Eq. 32 shows that if the relative risks for $x, w$, and $z$ are positively correlated, then the attributable fraction for disease risk within the population, is greater than would be estimated using the average relative risk, with estimates using the mean relative risk providing a lower bound. If $x$ and $z$ are negatively correlated then the $\geq \operatorname{sign}$ is be replaced by $\leq$.

Another quantity that might be considered is the expected value of the attributed fraction $1-1 / e^{\eta_{x}}$, that is $E\left[1-1 / e^{\eta_{x}}\right]=$ $1-E\left[1 / e^{\eta_{x}}\right]$. Because $1 / e^{\eta_{x}}$ is concave, Jensen's inequality gives,

$$
E\left[\frac{1}{e^{\eta_{x}}}\right] \geq \frac{1}{E\left[e^{\eta_{x}}\right]}
$$

and as a result,

$$
1-\frac{1}{E\left[e^{\eta_{x}}\right]} \geq 1-E\left[\frac{1}{e^{\eta_{x}}}\right]=E\left[1-\frac{1}{e^{\eta_{x}}}\right]
$$

If $e^{\eta_{x}}$ and $e^{\eta_{w}+\eta_{z}}$ are positively correlated, then Eqs. 34 and 32 indicate that $E\left[1-1 / e^{\eta_{x}}\right]$ will also bound Eq. 19. However, this would not be true if $e^{\eta_{x}}$ and $e^{\eta_{w}+\eta_{z}}$ were negatively correlated. 
medRxiv preprint doi: https://doi.org/10.1101/2021.12.24.21268368; this version posted December 25, 2021. The copyright holder for this preprint (which was not certified by peer review) is the author/funder, who has granted medRxiv a license to display the preprint in perpetuity.

All rights reserved. No reuse allowed without permission.

\section{Relation to other attributable fractions}

A recent study [18] with a proportion $p$ exposed to a virus, and an estimated relative risk $R$, reported an attribution fraction of $A=p(R-1) / R$. Here it is briefly outlined when this will approximate Eq. 19. Assume that $e^{\eta_{x}}, e^{\eta_{w}}$, and $e^{\eta_{z}}$ are uncorrelated, so that Eq. 16 simplifies to 17, that may be approximated as,

$$
A_{f} \simeq 1-\frac{1}{\frac{1}{n} \sum_{i=1}^{n} e^{\eta_{x_{i}}}}
$$

If we consider a proportion $p$ that are exposed with relative risk $R$, and a proportion $(1-p)$ that are unexposed, then using Eq. 35,

$$
\begin{aligned}
A_{f} & \simeq 1-\frac{1}{(1-p)+p R} \\
& =\frac{p(R-1)}{1+p(R-1)} \\
& =p\left(1-\frac{1}{R}\right)\left(\frac{R}{1+p(R-1)}\right) \\
& \simeq p\left(1-\frac{1}{R}\right)
\end{aligned}
$$

where the approximation in the final line follows if $R-1$ is small enough, as it often can be. A better approximation follows from the second line, where $p(R-1) \ll 1$ ensures that $A_{f} \simeq p(R-1)$. If $p \simeq 1$, then $A_{f} \simeq(R-1) / R$ as usual, as can be seen from the first or second line above.

\section{Supplementary tables and figures}
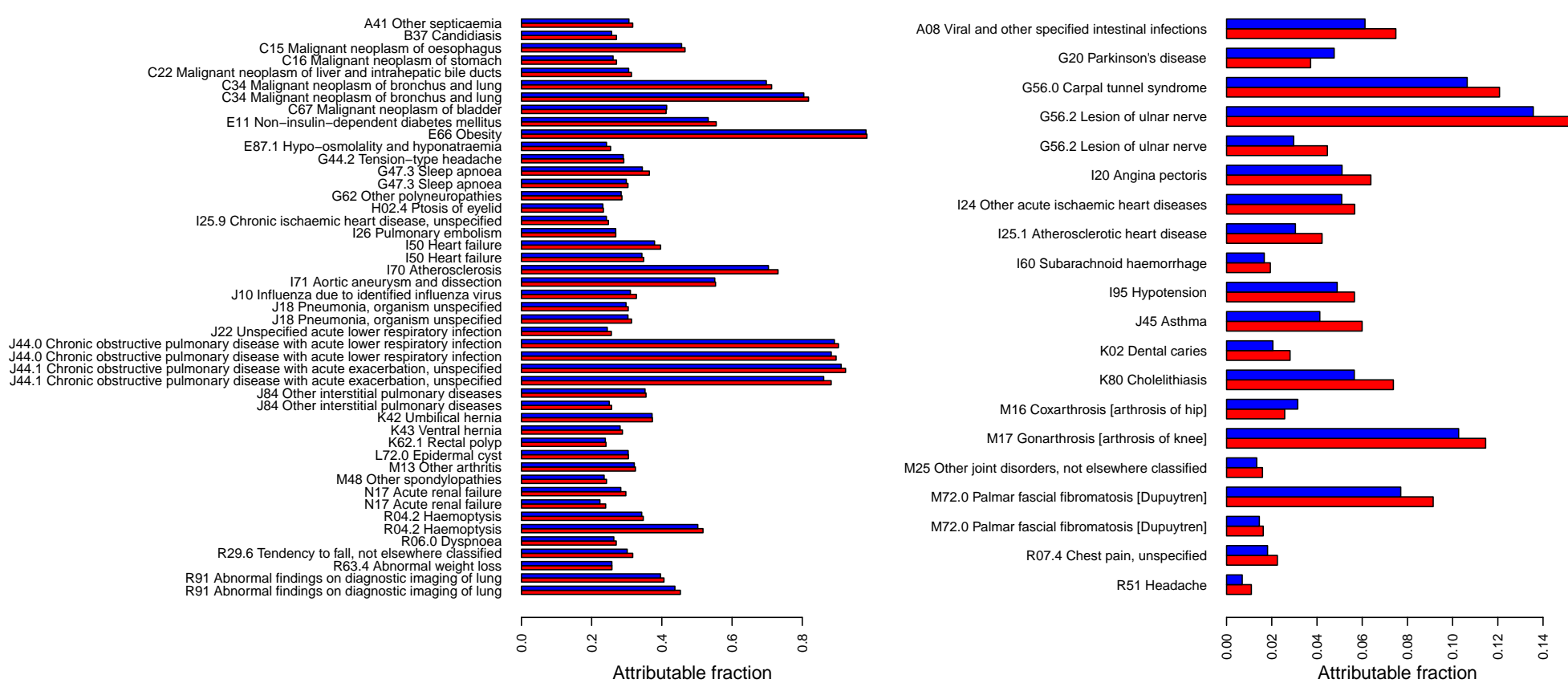

Figure 5: Attributable fractions $\left(A_{f}\right)$ for smoking and BMI, estimated with Eqs. 19 (red) and 18 (blue). Eq. 18 is equivalent to the WHO's $A_{f}$ [1]. Eq. 19 estimates causal associations. Left plot: diseases with the highest $25 \% A_{f}$. Right plot: diseases where Eqs. 18 and 19 differed the most. 
medRxiv preprint doi: https://doi.org/10.1101/2021.12.24.21268368; this version posted December 25, 2021. The copyright holder for this preprint (which was not certified by peer review) is the author/funder, who has granted medRxiv a license to display the preprint in

perpetuity.

All rights reserved. No reuse allowed without permission.

\begin{tabular}{llrrrrc}
\hline \multicolumn{1}{c}{ Disease } & Sex & N & $N_{A_{f}}$ & Rank & \multicolumn{1}{c}{$A_{f}$} & Rank $A_{f}$ \\
\hline E66 Obesity & $\mathrm{F}$ & 311 & 305 & 7 & 0.98 & 1 \\
\hline E11 Non-insulin-dependent diabetes mellitus & $\mathrm{M}$ & 206 & 94 & 38 & 0.45 & 2 \\
K42 Umbilical hernia & $\mathrm{F}$ & 297 & 120 & 32 & 0.40 & 3 \\
\hline G47.3 Sleep apnoea & $\mathrm{F}$ & 381 & 121 & 31 & 0.32 & 4 \\
I50 Heart failure & $\mathrm{M}$ & 359 & 111 & 34 & 0.31 & 5 \\
G62 Other polyneuropathies & $\mathrm{M}$ & 175 & 47 & 66 & 0.27 & 6 \\
M13 Other arthritis & $\mathrm{M}$ & 215 & 57 & 53 & 0.27 & 7 \\
I50 Heart failure & $\mathrm{F}$ & 280 & 74 & 44 & 0.26 & 8 \\
I26 Pulmonary embolism & $\mathrm{F}$ & 836 & 194 & 16 & 0.23 & 9 \\
G47.3 Sleep apnoea & $\mathrm{M}$ & 776 & 172 & 21 & 0.22 & 10 \\
J22 Unspecified acute lower respiratory infection & $\mathrm{F}$ & 1506 & 326 & 6 & 0.22 & 11 \\
J10 Influenza due to identified influenza virus & $\mathrm{F}$ & 211 & 43 & 73 & 0.20 & 12 \\
I25.9 Chronic ischaemic heart disease, unspecified & $\mathrm{M}$ & 254 & 51 & 59 & 0.20 & 13 \\
M81 Osteoporosis without pathological fracture & $\mathrm{F}$ & 946 & 191 & 17 & 0.20 & 14 \\
\hline
\end{tabular}

Table 4: BMI only: Sex indicates diseases in males (M) or females (F), $N$ are total cases, $N_{A_{f}}$ are cases attributed to BMI, $A_{f}$ is the attributable fraction for deviations from the mid-tertile of BMI. For obesity, unsurprisingly $A_{f} \simeq 1$. Reporting errors may have prevented $A_{f}=1$ for obesity.

\begin{tabular}{|c|c|c|c|c|c|c|}
\hline Disease & Sex & $\mathrm{N}$ & $N_{A_{f}}$ & Rank & $A_{f}$ & Rank $A_{f}$ \\
\hline J44.1 Chronic obstructive pulmonary disease with acute exacerbation, unspecified & $\mathrm{F}$ & 209 & 189 & 28 & 0.90 & 1 \\
\hline J44.0 Chronic obstructive pulmonary disease with acute lower respiratory infection & $\mathrm{F}$ & 416 & 368 & 10 & 0.89 & 2 \\
\hline J44.0 Chronic obstructive pulmonary disease with acute lower respiratory infection & M & 417 & 364 & 12 & 0.87 & 3 \\
\hline J44.1 Chronic obstructive pulmonary disease with acute exacerbation, unspecified & M & 261 & 227 & 21 & 0.87 & 4 \\
\hline C34 Malignant neoplasm of bronchus and lung & M & 1018 & 811 & 2 & 0.80 & 5 \\
\hline I70 Atherosclerosis & M & 156 & 115 & 50 & 0.74 & 6 \\
\hline C34 Malignant neoplasm of bronchus and lung & $\mathrm{F}$ & 996 & 702 & 3 & 0.70 & 7 \\
\hline I71 Aortic aneurysm and dissection & M & 402 & 230 & 20 & 0.57 & 8 \\
\hline R04.2 Haemoptysis & M & 314 & 134 & 43 & 0.43 & 9 \\
\hline C15 Malignant neoplasm of oesophagus & M & 473 & 194 & 26 & 0.41 & 10 \\
\hline R91 Abnormal findings on diagnostic imaging of lung & M & 358 & 145 & 37 & 0.41 & 11 \\
\hline C67 Malignant neoplasm of bladder & M & 1063 & 414 & 8 & 0.39 & 12 \\
\hline R91 Abnormal findings on diagnostic imaging of lung & $\mathrm{F}$ & 372 & 131 & 45 & 0.35 & 13 \\
\hline J84 Other interstitial pulmonary diseases & $\mathrm{F}$ & 177 & 53 & 83 & 0.30 & 14 \\
\hline B37 Candidiasis & M & 173 & 48 & 90 & 0.28 & 15 \\
\hline J84 Other interstitial pulmonary diseases & M & 234 & 64 & 72 & 0.27 & 16 \\
\hline J90 Pleural effusion, not elsewhere classified & M & 524 & 137 & 41 & 0.26 & 17 \\
\hline R04.2 Haemoptysis & $\mathrm{F}$ & 239 & 62 & 75 & 0.26 & 18 \\
\hline G56.2 Lesion of ulnar nerve & $\mathrm{F}$ & 222 & 52 & 85 & 0.23 & 19 \\
\hline K92.0 Haematemesis & M & 156 & 36 & 102 & 0.23 & 20 \\
\hline C22 Malignant neoplasm of liver and intrahepatic bile ducts & M & 171 & 39 & 97 & 0.23 & 21 \\
\hline R29.6 Tendency to fall, not elsewhere classified & M & 182 & 40 & 94 & 0.22 & 22 \\
\hline R06.0 Dyspnoea & M & 650 & 142 & 39 & 0.22 & 23 \\
\hline J18 Pneumonia, organism unspecified & M & 3011 & 645 & 4 & 0.21 & 24 \\
\hline C16 Malignant neoplasm of stomach & M & 260 & 55 & 79 & 0.21 & 25 \\
\hline H02.0 Entropion and trichiasis of eyelid & M & 337 & 68 & 69 & 0.20 & 26 \\
\hline K62.1 Rectal polyp & M & 1143 & 231 & 18 & 0.20 & 27 \\
\hline G44.2 Tension-type headache & $\mathrm{F}$ & 152 & 31 & 110 & 0.20 & 28 \\
\hline
\end{tabular}

Table 5: Smoking only: Sex indicates diseases in males (M) or females (F), $N$ are total cases, $N_{A_{f}}$ are cases attributed to smoking, $A_{f}$ is the attributable fraction. 
medRxiv preprint doi: https://doi.org/10.1101/2021.12.24.21268368; this version posted December 25, 2021. The copyright holder for this preprint (which was not certified by peer review) is the author/funder, who has granted medRxiv a license to display the preprint in perpetuity.

All rights reserved. No reuse allowed without permission.

\begin{tabular}{|c|c|c|c|c|c|c|}
\hline Disease & Sex & $\mathrm{N}$ & $N_{A_{f}}$ & Rank & $A_{f}$ & Rank $A_{f}$ \\
\hline N81 Female genital prolapse & $\mathrm{F}$ & 4199 & -788 & 1 & -0.190 & 1 \\
\hline L72.0 Epidermal cyst & $\mathrm{F}$ & 525 & -96 & 13 & -0.180 & 2 \\
\hline N41 Inflammatory diseases of prostate & M & 572 & -103 & 12 & -0.180 & 3 \\
\hline S02 Fracture of skull and facial bones & M & 355 & -36 & 23 & -0.100 & 4 \\
\hline K40 Inguinal hernia & $\mathrm{F}$ & 493 & -49 & 20 & -0.100 & 5 \\
\hline R19.8 Other specified symptoms and signs involving the digestive system and abdomen & $\mathrm{F}$ & 302 & -30 & 25 & -0.098 & 6 \\
\hline M20.1 Hallux valgus (acquired) & $\mathrm{F}$ & 2875 & -280 & 2 & -0.097 & 7 \\
\hline S76.1 Injury of quadriceps muscle and tendon & M & 215 & -20 & 26 & -0.095 & 8 \\
\hline R79 Other abnormal findings of blood chemistry & M & 1905 & -169 & 5 & -0.089 & 9 \\
\hline D04 Carcinoma in situ of skin & M & 201 & -16 & 27 & -0.081 & 10 \\
\hline K52.9 Non-infective gastro-enteritis and colitis, unspecified & M & 1061 & -76 & 15 & -0.071 & 11 \\
\hline S82 Fracture of lower leg, including ankle & $\mathrm{F}$ & 2163 & -110 & 10 & -0.051 & 12 \\
\hline
\end{tabular}

Table 6: BMI only: Diseases with the strongest protective associations, ranked by attributable fraction $\left(A_{f}\right)$. Sex indicates diseases in males (M) or females (F), $N$ are total cases, $N_{A_{f}}$ are the number of cases attributed to BMI.

\begin{tabular}{llrrrrc}
\hline \multicolumn{1}{c}{ Disease } & $\mathrm{Sex}$ & $\mathrm{N}$ & \multicolumn{1}{c}{$N_{A_{f}}$} & Rank & \multicolumn{1}{c}{$A_{f}$} & Rank $A_{f}$ \\
\hline G20 Parkinson's disease & $\mathrm{F}$ & 152 & -31 & 18 & -0.200 & 1 \\
\hline D03 Melanoma in situ & $\mathrm{M}$ & 272 & -44 & 14 & -0.160 & 2 \\
G20 Parkinson's disease & $\mathrm{M}$ & 289 & -41 & 15 & -0.140 & 3 \\
C43 Malignant melanoma of skin & $\mathrm{M}$ & 723 & -86 & 8 & -0.120 & 4 \\
S52 Fracture of forearm & $\mathrm{M}$ & 687 & -74 & 9 & -0.110 & 5 \\
K31.7 Polyp of stomach and duodenum & $\mathrm{F}$ & 870 & -89 & 7 & -0.100 & 6 \\
C54 Malignant neoplasm of corpus uteri & $\mathrm{F}$ & 878 & -70 & 12 & -0.080 & 7 \\
R79 Other abnormal findings of blood chemistry & $\mathrm{M}$ & 1905 & -150 & 4 & -0.079 & 8 \\
R19.5 Other fecal abnormalities & $\mathrm{F}$ & 544 & -32 & 17 & -0.059 & 9 \\
N40 Hyperplasia of prostate & $\mathrm{M}$ & 3928 & -214 & 3 & -0.055 & 10 \\
C61 Malignant neoplasm of prostate & $\mathrm{M}$ & 5800 & -304 & 1 & -0.052 & 11 \\
K31.7 Polyp of stomach and duodenum & $\mathrm{M}$ & 300 & -15 & 21 & -0.051 & 12 \\
\hline
\end{tabular}

Table 7: Smoking only: Diseases with the strongest protective associations, ranked by attributable fraction $\left(A_{f}\right)$. Sex indicates diseases in males (M) or females (F), $N$ are total cases, $N_{A_{f}}$ are the number of cases attributed to smoking. 
medRxiv preprint doi: https://doi.org/10.1101/2021.12.24.21268368; this version posted December 25, 2021. The copyright holder for this preprint (which was not certified by peer review) is the author/funder, who has granted medRxiv a license to display the preprint in perpetuity.

All rights reserved. No reuse allowed without permission.

$X$ Diseases of the respiratory system $(\mathrm{N}=13)$

VIII Symptoms, signs and abnormal clinical and laboratory findings, not elsewhere classified $(\mathrm{N}=18)$

I Certain infectious and parasitic diseases $(\mathrm{N}=6)$

II Neoplasms $(\mathrm{N}=20)$

$\mathrm{XI}$ Diseases of the digestive system $(\mathrm{N}=19)$

XIII Diseases of the musculoskeletal system and connective tissue $(\mathrm{N}=10)$

XII Diseases of the skin and subcutaneous tissue $(\mathrm{N}=12)$

IX Diseases of the circulatory system $(\mathrm{N}=17)$

XIV Diseases of the genitourinary system $(\mathrm{N}=11)$

VI Diseases of the nervous system $(\mathrm{N}=6)$

VII Diseases of the eye and adnexa $(\mathrm{N}=7)$

$\mathrm{XIX}$ Injury, poisoning and certain other consequences of external causes $(\mathrm{N}=8)$
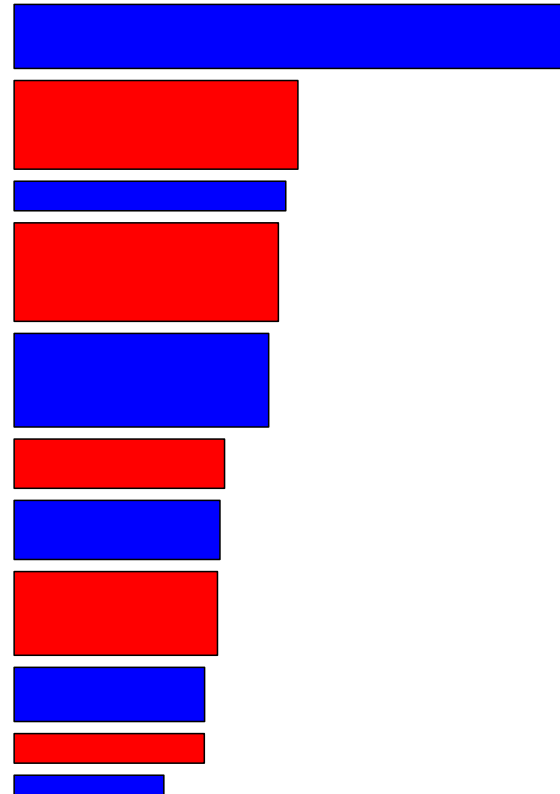

\section{$\square$}

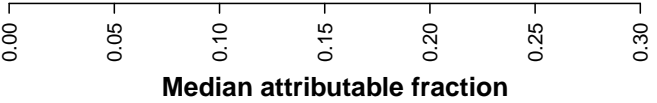

Attribution fractions for smoking (existing UK Biobank population, versus if all never smoked)

XIII Diseases of the musculoskeletal system and connective tissue ( $N=24)$

VI Diseases of the nervous system $(\mathrm{N}=5)$

$X$ Diseases of the respiratory system $(\mathrm{N}=6)$

IX Diseases of the circulatory system $(\mathrm{N}=12)$

XIV Diseases of the genitourinary system $(\mathrm{N}=12)$

XII Diseases of the skin and subcutaneous tissue $(\mathrm{N}=7)$
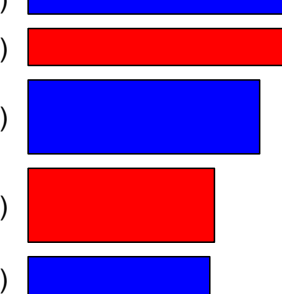

$\mathrm{XI}$ Diseases of the digestive system $(\mathrm{N}=19)$
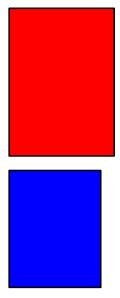

II Neoplasms $(\mathrm{N}=15)$

$\mathrm{XIX}$ Injury, poisoning and certain other consequences of external causes $(\mathrm{N}=7)$

VIII Symptoms, signs and abnormal clinical and laboratory findings, not elsewhere classified $(\mathrm{N}=11)$

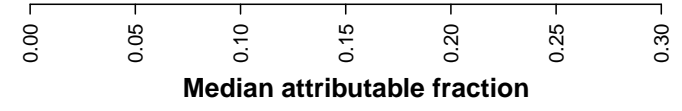

\section{Attribution fractions for BMI (existing UK Biobank population, versus if all were mid-tertile BMI)}

Figure 6: Median attributable fractions for each ICD-10 chapter with at least 5 diseases where $A_{f}>0.2$. Bar widths are proportional to the number of diseases in each chapter. 